\title{
The Application of Comparative Teaching Method in the Course of Aeronautic Equipment Storehouse Mold Proof
}

\author{
Li Li, Zhao Hongqiang, Shi Yumin'Zhang Suqin, Wang Jiongkun, Guo Xingxiang, Li Kun
}

\author{
Naval Aviation University Qingdao Branch, Qingdao, Shangdong, 266041, China \\ Corresponding author.Email:qdxqlixia@163.com
}

\begin{abstract}
In order to improve the class teaching effect, cultivate the students' ability of comparison, analysis and identification, and promote their comprehensive and systematic understanding of the problems, the comparative teaching method is adopted in the course of teaching. Combined with the characteristics of the course of "mold proof technology of aeronautic equipments storehouse", such as strong theoretical basis, complex knowledge points, complicated and meticulous operation steps of the experimental part, and difficult verification of experimental results, we have adopted the method of "grasping common features, finding differences and distinguishing advantages and disadvantages" in the teaching course. The practice proves that the comparative teaching method is helpful for students to think about the teaching contents and analyze them comprehensively and deeply grasp its regularity, so as to achieve a rational understanding, which plays an important supporting role in improving the management level of aeronautic equipments safeguard of students. Keywords: comparative teaching method; aeronautic equipments safeguard; storehouse mold
\end{abstract}

\section{INTRODUCTION}

Aeronautic equipments are the material basis for the Naval Aviation Forces to complete various operations and training tasks. The insufficient quantity or quality of aeronautic equipments stored in the aeronautic equipments storehouse may cause the aircraft to stop flying, reduce the good rate of monthly aeronautic equipments support, and directly affect the formation of the combat power of the Aviation Forces, so it is difficult to ensure that can complete the military training tasks smoothly by the Aviation Forces. Due to the particularity of the task, the environmental and climatic characteristics of "three highs" (i.e. high temperature, high humidity and high salt, and even high sunshine in some places all the year round, commonly known as "four highs") are easy to cause the breeding and spreading of the mold in the storage, resulting in the serious degradation of the performance of the equipments. In order to ensure the quality and quantity of the equipments in the storage, the "none of four" about aeronautic equipments (one of which is " there is no corrosion and mildew") must be done. In the personnel training program of aeronautic equipments management specialty at the level of vocational and technical education for noncommissioned officers, the basic course of aeronautic equipments storage mold proof technology is set up. Students are required to be able to identify the main types of mold in aeronautic equipments storage through the learning of this course, describe the micro morphological structure and macro colony characteristics of the mold, and name the asexual and sexual reproduction and spore types of the mold, the growth conditions of the mold in nature, the growth and propagation rules and characteristics of mold, induce the multiple influencing factors of the mold growth, elaborate the physical and chemical control methods of the mold, and conclude the comprehensive control measures of the mold in aeronautic equipments storehouse, so as to finally achieve the course purpose of cultivating students' ability of mold proof and good professional quality in aeronautic equipments storehouse.

\section{THE CONNOTATION AND SIGNIFICANCE OF COMPARATIVE TEACHING METHOD}

\subsection{The connotation of comparative teaching method}

The comparative teaching method is a kind of teaching method which focuses on thinking discrimination and can determine the similarities and differences between teaching contents. In the process of teaching, in order to achieve the expected teaching purpose, according to certain comparative standards, teachers first select the course contents that are related to each other and different from each other, and then make a horizontal comparative analysis of these contents to find out the similarities and differences, which can make students have associative memory of knowledge and improve the teaching effect ofthe course[1]. 


\subsection{The significance of comparative teaching method}

The application of comparative teaching method is not only conducive to the cultivation of students' ability of independent thinking and independent learning, but also to the cultivation of students' ability of in-depth analysis and active exploration, as well as to the cultivation of students' ability of drawing inferences from one instance and making a comprehensive study. In order to better let the students master the obscure theoretical knowledge in the course, the teacher can carefully select relevant knowledge points for comprehensive analysis and comparison, explain the similarities and differences of knowledge from multiple sides, guide the students to carry out series and parallel connection of knowledge step by step, and realize the improvement of students' ability. The reasonable application of this teaching method can greatly stimulate the students' learning interest in the teaching process, make the students change from "want me to learn" to "I want to learn", make full use of the students' subjective initiative in learning, so as to further strengthen post capacity of the students ${ }^{[2,3]}$. In order to ensure the smooth and complete use of comparative teaching method in the teaching process, the implementation can be organized according to the steps shown in Fig.1.

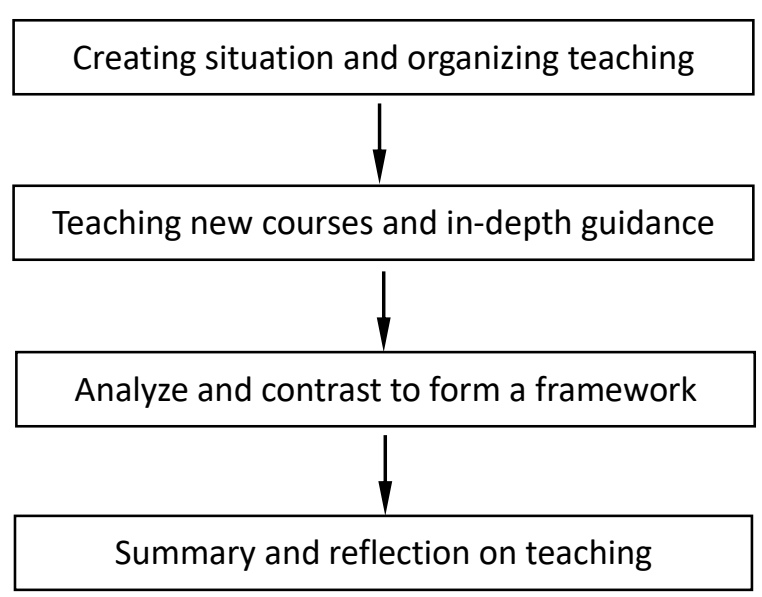

Fig.1. Organization process of comparative teaching method

\section{A CASE STUDY ON THE APPLICATION OF COMPARATIVE TEACHING METHOD}

From the perspective of thinking training, there are two common methods of comparative teaching method: seeking the same comparison method and seeking different comparison method. Among them, the method of seeking the same refers to comparing the knowledge which has similarities on the surface but has differences in essence, so that students can make use of the connection between knowledge, seek differences in the same, seek the same in the different, deepen understanding, enhance memory, and cultivate students' ability to distinguish things. The method of seeking difference means that there are differences between things, one is the comparison between different things, the other is the comparison between two different aspects of the same thing, that is, put two opposite concepts together, analyze their characteristics from different sides, form a sharp contrast, and deepen the impression of students. Generally, these two methods cooperate with each other and complement each other, so as to draw an analogy of knowledge.

\subsection{The different ways of reproduction have various characteristic of spores}

For example, when we explain the knowledge points of asexual propagation of the mold, we tell that asexual propagation includes five different kinds of asexual spores, including sporangia spore, conidia, arthrospores, chlamydospores and thallospore. Different kinds of mold produce different kinds of spores when they propagate, Rhizopus and Mucor can produce sporangia, Aspergillus and Penicillium can produce conidia, and Geotrichum candidum can produce arthrospores; The same kind of mold can produce different kinds of spores in different growth environment. For example, Rhizopus usually produces sporangia in asexual propagation stage, while the "yeast cell" formed in liquid medium belongs to the spore. The asexual spore is formed by the bud of mother cell, in which the hypha cell produces small protuberances similar to germination, which are formed by the overflow and contraction of cell wall from the mother cell, then it becomes a kind of spherical spore. It can be found that the same point of the five kinds of spores is that the chromosome multiples are haploid, the different points include the endogenesis or exogenesis of spores, and the formation characteristics also include several ways, such as the hypha top expanding and breaking or the hypha breaking at the diaphragms. The spore morphology also has many shapes, such as near circle, tube and ball. The above contents are listed in the table, we make a specific comparison of the characteristics of each spore, which helps students to clearly identify the knowledge points and truly internalize the knowledge they have learned into their post ability ${ }^{[4]}$.

\subsection{The differences in transport materials have outstanding absorption characteristics}

For instance, because there is no special organ for the storage mold to absorb nutrients, the nutrition intake mainly depends on the whole cell surface. According to the characteristics of substance transport process, there are four main ways of nutrients entering cells: simple diffusion, facilitated diffusion, active transport and group transfer. In order to deepen and consolidate the students understanding and memory of the difficult knowledge, 
firstly, four different absorption modes are shown to the students through animation demonstration, and then the characteristics of each mode are explained in detail. Finally, whether there is specific carrier protein, transport speed, transport direction of substances, concentration inside and outside cells, transport molecules are specific or not, and whether they need to the energy consumption and the change of material structure after transportation are analyzed and compared in tables. At the same time, in the teaching process, it also emphasizes the application of seeking difference in the same and seeking the same in the difference. For example, the concentration of substance transport is from high to low, the former does not need transport carrier, and the latter needs carrier protein, but finally reaches the same concentration in and out of the transport substance cell; active transport and group transfer need to consume energy in the transport process, but before and after transportation, the former's substance structure remains unchanged, while the latter's structure changes. Therefore, through this all-round and multi angle comparative analysis, students can greatly enhance the three-dimensional cognition of knowledge, which is conducive to the digestion and absorption of curriculum content and ability transformation ${ }^{[5]}$.

\subsection{The continuous growth periods have the different reproduction law}

For another example, the growth and reproduction law of storage mold is also the key and difficult content of this course. According to the different growth rate of mold, the curve can be divided into four periods, i.e. delay period, logarithm period, stability period and decay period. Why will the mold inoculated into the new medium have a delay period? What are the characteristics of each period? Is the growth rate constant of the delay period and the stable period basically zero? In order to solve the above questions, each question of the four periods is analyzed by the comparative teaching method. The growth rate constant of the delay period is zero, because the spores do not grow basically, and because the spores of the death number and the newly proliferated number are almost equal, the growth rate constant of the stable period on the curve is zero. After comparing the contents of each part, in order to facilitate the students' memory, the characteristics of the four periods are summarized respectively. The delay period can be summarized as follows: slow division and active metabolism; the characteristics of the logarithmic period can be summarized as follows: vigorous metabolism and stable speed; the characteristics of the stable period can be summarized as follows: increase and decrease offset, dynamic balance, and delay period can be described as increase rather than decrease, and the number plummets. The highly condensed and multi-dimensional comparison of the course content has brought new learning experience to the students and greatly inspired their enthusiasm for learning ${ }^{[6]}$.

\subsection{The combination of physical and chemical methods has significant effect of mold proof}

The final goal of this course is to prevent mold in aeronautic equipments storage. From the identification of mold species to the growth conditions of mold, from the mode of mold reproduction to the growth and propagation rules and influence factors of mold, these theoretical knowledge are all the preparation for the study of comprehensive treatment measures of aeronautic equipments storage mold. There are two ways to control mold: physical method and chemical method. In the storehouse, two methods often complement each other and play an irreplaceable role. In order to let the students master the characteristics of the two methods, the physical and chemical methods are compared and optimized in the teaching process. The physical method used in the storehouse is usually the ultraviolet lamp irradiation method. First, the students will be told the principle of ultraviolet sterilization, and then explain how to determine the quantity of ultraviolet lamp installation according to the storehouse area and the wattage of the lamp, and how long the ultraviolet lamp needs to be continuously irradiated after it is turned on, including the precautions to use the ultraviolet lamp, etc. Finally, the students will learn the effect of ultraviolet on the growth of mold through practical teaching. The method of chemical control of mold is also in accordance with the order of "sterilization principle - use method - precautions", which is in turn compared with physical sterilization method. For example, there are usually four ways to use fungicide, and the spraying method is selected in the storehouse. First, the fungicide should be prepared according to the storehouse area and the mold growth of the equipments in the storehouse. Then, taking the mold research results of the aeronautic equipments storehouse as an example, they are introduced respectively through the real experimental data about the amount of the fungicide, and when to spray fungicide, and how long the doors and windows are closed after spraying and so on. Finally, combining with the growth and propagation law of mold, let students distinguish physical method which is often used to prevent the front stage of mold growth. Once it is found that the growth of mold is close to the logarithmic period and there is an irresistible momentum, the method of spraying fungicide must be taken immediately. Only when the two methods are combined the effect of both treatment and specimen be achieved. The comparison teaching method makes a concise comparison of the abstract and obscure teaching contents, enhances the comprehensive analysis ability of the students, improves the efficiency of the class teaching, and lays a solid foundation for the students to competent the post of aeronautic equipments store keeper $^{[7]}$.

\section{CONCLUSION}

The comparative teaching method is a thinking process and 
method for teachers to distinguish and determine the similarities and differences between teaching contents in teaching practice. In the process of teaching, teachers should first extract the knowledge points suitable for comparative teaching through the integration of teaching contents, then guide the students to think through the whole method, see the essence through the phenomenon, carry out in-depth comparison, select the appropriate comparison method, lead the students to try and explore continuously, stimulate the students' sense of participation and competition, and improve the students' enthusiasm and initiative in learning mobility. The reasonable application of comparative teaching method can cultivate students' ability of knowledge integration and application, enable students to learn knowledge in a relaxed and pleasant learning atmosphere, improve teaching efficiency, enhance learning effect, and enable students to get twice the result with half the effort.

\section{ACKNOWLEDGMENT}

This paper was supported by education reform project of Naval Aviation University.

\section{REFERENCES}

[1] Chen Mei. On the teaching method of cross field comparison [J]. Basic education forum, 2019, 4:25-26.

[2] Wang Li. The application of comparative teaching method in the teaching of real variable function - an analysis of comparative teaching with mathematical analysis [J]. Education and teaching forum, 2019, 9:180-182.

[3] Chao Lina. Research on comparative teaching method and metaphor teaching method in high school biology concept teaching [M]. Nanchang: Jiangxi Normal University, 2014.

[4] Li Cheng. An analysis of the application of comparative teaching method in the course of fundamentals of computer application [J]. Computer knowledge and technology, 2019,15 (26): 140-141.

[5] Mu fengjuan, Hu Xiu. Application of comparative teaching method in the teaching of dendrology [J]. China forestry education, 2019,5 (3): 40-43.

[6] Zhou Hui. Application of comparative teaching method in teaching angina and myocardial infarction [J]. Education modernization, 2019,3 (23): 103-104.

[7] Gao Xiang, Ye Xiangqun, etc. Application of comparative teaching method in the teaching of chemical engineering principle experiment [J]. Laboratory research and exploration, 2018,11 (11): 215-218. 